\title{
Four Stage Encryption Generalizations: Partitioned Output Cryptosystem
}

\author{
Sangapu Venkata Appaji \\ Assistant Professor \\ Department of Information Technology, \\ Gokaraju Ranga raju Institute of Engineering and \\ Technology, Hyderabad, India
}

\author{
Gomatam V S Acharyulu, Ph.D. \\ Professor \\ Department of Computer Science and Engineering \\ Geethanjali College of Engineering and \\ Technology, Hyderabad, India
}

\begin{abstract}
The Four Stage Encryption System introduced by Acharyulu and Appaji provides secrecy even when the attacker has some samples of plain texts and their corresponding cipher texts obtained with the same key, because the cipher text generated each time, for the same plain text with the same key, is different. In other words, the system is secure against adaptive chosen-plain text attack. Another interesting feature is that even the size of the cipher text, for given plain text under a given key, is unpredictable. In this paper the four stage encryption system is generalized as Generated Partitioned Output Crypto System and some variations that can be taken up are suggested. A pure mathematical system called Partitioned Output Crypto System is introduced as a further step of generalization in order to study the properties of the system. A correlation with the existing systems is taken up as a part of the study of the properties. It's observed that the system shares the properties of multifunction.
\end{abstract}

\section{General Terms}

Cryptography, Cryptanalysis, Security.

\section{Keywords}

Block Ciphers, Cryptosystems, Ciphers, Multi-functions, Partitions.

\section{INTRODUCTION}

A cryptosystem [5], [4] is a quintet (M, C, K, e, d), where [5], [4]

1. $\mathrm{P}$ is a finite set, called message space (plain texts).

2. C is a finite set, called cryptotext space (cyptotexts).

3. $\mathrm{K}$ is a finite set, called key space.

4. $\mathrm{e}$ is a function, $\mathrm{e}: \mathrm{K} \times \mathrm{M} \rightarrow \mathrm{C}$, whose domain $\mathrm{K} \times \mathrm{M}$ is the set of all order pairs $(\mathrm{k}, \mathrm{m})$ where $\mathrm{k}$ and $\mathrm{m}$ are elements of $\mathrm{K}$ and $\mathrm{M}$ respectively.

5. $\mathrm{d}$ is a function, $\mathrm{d}: \mathrm{K} \times \mathrm{C} \rightarrow \mathrm{M}$, whose domain $\mathrm{K} \times \mathrm{C}$ is the set of all ordered pairs $(\mathrm{k}, \mathrm{c})$ where $\mathrm{k}$ and $\mathrm{c}$ are elements of $\mathrm{K}$ and $\mathrm{C}$ respectively, such that $\mathrm{d}(\mathrm{k}, \mathrm{e}(\mathrm{k}, \mathrm{m}))=\mathrm{m}$ for any $\mathrm{m}$ in P. It is sometimes convenient to write the dependence on $\mathrm{k}$ as subscript. Then for each $\mathrm{k}$ in $\mathrm{K}$, we get a pair of functions $\mathrm{e}_{\mathrm{k}}: \mathrm{M} \rightarrow \mathrm{C}$ and $\mathrm{d}_{\mathrm{k}}: \mathrm{C} \rightarrow \mathrm{M}$ such that $\mathrm{d}_{\mathrm{k}}\left(\mathrm{e}_{\mathrm{k}}(\mathrm{m})\right)=$ $\mathrm{m}$ for all $\mathrm{m}$ in $\mathrm{M}$.

In order for (M, C, K, e, d) to be a successful cipher, it must have the following properties:

1. For any key $\mathrm{k}$ in $\mathrm{K}$, and plain text $\mathrm{m}$ in $\mathrm{M}$, it must be easy to compute the cipher text $\mathrm{e}_{\mathrm{k}}(\mathrm{m})$.

2. For any key $\mathrm{k}$ in $\mathrm{K}$, and cipher text $\mathrm{c}$ in $\mathrm{C}$, it must be easy to compute $\mathrm{d}_{\mathrm{k}}(\mathrm{c})$.
3. Given one or more cipher texts, $c_{1}, c_{2}, \ldots, c_{n}$ in $C$ encrypted using the key $\mathrm{k}$ in $\mathrm{K}$, it must be very difficult to compute any of the plain texts $d_{k}\left(c_{1}\right), d_{k}\left(c_{2}\right), \ldots, d_{k}\left(c_{n}\right)$ without the knowledge of $\mathrm{k}$.

There is a fourth property that is desirable, although it is more difficult to achieve.

4. Given one or more pairs of plaintexts and the subsequent cipher texts $\left(\mathrm{p}_{1}, \mathrm{c}_{1}\right),\left(\mathrm{p}_{2}, \mathrm{c}_{2}\right), \ldots,\left(\mathrm{p}_{\mathrm{n}}, \mathrm{c}_{\mathrm{n}}\right)$ it must be hard to decrypt any cipher text $\mathrm{c}$ that is not in the given list without knowing $\mathrm{k}$. This is known as security against a chosen plaintext attack.

Four stage encryption system [1], [2] has some interesting features. In order to give further scope of work, this system is generalized the system is called generated output cryptosystem (GPOCS). A few variants of this generalized system were given in the examples. A mathematical structure called partitioned output cryptosystem is also suggested in order to study the properties of system.

Manes and Benson studied sum ordered partial rings [3], [8], [9], [10] in an abstract way and showed that many properties enjoyed by the set of all partialfuctions from $D$ into $D$ is denoted by $\operatorname{Pfn}(\mathrm{D}, \mathrm{D})$, the set of all multifunctions from $\mathrm{D}$ into $\mathrm{D}$ is denoted by $\operatorname{Mfn}(\mathrm{D}, \mathrm{D})$, and the set of all multisets is denoted by $\operatorname{Mset}(\mathrm{D}, \mathrm{D})$ in the context of representing recursive programs as matrices of partial functions. They found that every so-ring contains an inverse sub semi group of inversible elements. It is interesting to note the properties of $\operatorname{Mfn}(\mathrm{D}, \mathrm{E})$, the set of all multifunctions of $\mathrm{D}$ into $\mathrm{E}$, in the context of Cryptosystems, as they share some properties. By a multifunction from a set $\mathrm{D}$ into a set $\mathrm{E}$, we mean a function $f$ : $\mathrm{D} \rightarrow 2^{\mathrm{E}}$, where $\mathrm{D}$ is the domain of $f$ and for any given element $\mathrm{d}$ of $\mathrm{D}, f(\mathrm{~d})$ is a subset of $\mathrm{E}$, possibly empty. The set of all multifunctions from $D$ into $E$ is denoted by $\operatorname{Mfn}(D, E)$. one can easily realize that multifunction is a relation from $\mathrm{D}$ in to E.

\section{RELATED WORK}

The four stage encryption and decryption are given as follows [2], [12].

\subsection{Four Stage Encryption Algorithm}

Let $\mathrm{A}$ be the input alphabet, a finite ordered set of symbols comprising the language [2].

Let $\mathrm{K}=\mathrm{K}_{0} \quad \mathrm{~K}_{1} \quad \mathrm{~K}_{2}$ be the three stage key, where each $\mathrm{Ki}$ is an element of $\mathrm{A}^{+}$, the set of non-null strings of $\mathrm{A}$. Let $\alpha \in \mathrm{A}^{+}$be the string to be encrypted. The following steps encrypt $\alpha$ into $\beta$. 
Step 1: Construct $Z=\cup_{a \in A} a A^{\text {index }\left(k_{0}(\text { index }(a))\right.}$ and let $\mathrm{m}$ $=|Z|=|\mathrm{A}|\left(\sum a \in \mathrm{A}\right.$ index $\left(\mathrm{K}_{0}(\operatorname{index}(\mathrm{a}))\right)$, where $\mathrm{K}_{0}(\mathrm{i})$ stands for the $\left(i \bmod \left|K_{0}\right|\right)^{\text {th }}+1$ letter of $K_{0}$.

Step 2: Construct a permutation matrix $\mathrm{M}$ of order $\mathrm{m}$ from $\mathrm{K}_{1}$ as follows: Let $\mathrm{i}=1$ and $\mathrm{p}=1$

While $\mathrm{j} \leq m$ do

$$
\text { Let } \mathrm{i} \text { be the index of } \mathrm{p}^{\text {th }} \text { letter of } \mathrm{K}_{1} \text {. }
$$

If $\mathrm{i}$ is a repetition, increment until an unrepeated number is obtained.

Let $\delta_{\mathrm{i}}$ be a column vector of size $\mathrm{m}$ with $\mathrm{i}^{\text {th }}$ entry as a 1 and all other entries as 0 .

Let $\delta_{i}$ be the $\mathrm{j}^{\text {th }}$ column of the matrix M.

Increment $\mathrm{j}$.

Let $\mathrm{p}=\left(\mathrm{j} \bmod \left|\mathrm{K}_{1}\right|\right)+1$.

End while.

Step 3: Let $\mathrm{Y}=\mathrm{ZM}$. ( $\mathrm{Y}$ is a permutation of $\mathrm{Z}$ )

Step 4: Construct an n-partition of $m$ using $K_{2}$ as follows: Let $\mathrm{m}_{1}, \mathrm{~m}_{2}, \ldots, \mathrm{m}_{\mathrm{n}}$ be the indices of the letters of the word $\mathrm{K}_{2}$ taken in round robin pattern. Let $\mathrm{q}=\mathrm{m}_{1}+\mathrm{m}_{2}+\cdots+$ $\mathrm{m}_{\mathrm{n}}$ and $\mathrm{r}=|\mathrm{Z}| \operatorname{div} \mathrm{q}$ (quotient of $|\mathrm{Z}|$ divided by $\mathrm{q}$ ). Partition $\mathrm{Y}$ by taking in order the $\mathrm{r} . \mathrm{m}_{1}, r . \mathrm{m}_{2}, \ldots, \mathrm{r} . \mathrm{m}_{\mathrm{n}-1}$ the remaining elements as the last block. Let these blocks be $\mathrm{Y}_{1}, \mathrm{Y}_{2}, \ldots, \mathrm{Y}_{\mathrm{n}}$.

Step 5: For each $a \in \mathrm{A}$, a random function $f_{a}: \mathrm{N} \rightarrow \mathrm{Y}_{\mathrm{i} a}$ is generated, where i $a$ is the $\operatorname{index} f_{a}$ in $\mathrm{A}$.

Step 6: Let the encrypted text $\beta$ be null string.

Step 7: For each letter a of the plain text $\alpha$, do

Find $\mathrm{t}=\mathrm{o}(a, \alpha)$, the order of occurrence of $a$ in $\alpha$. Encrypt a by $f_{a}(\mathrm{t})$.

Concatenate $f_{a}(t)$ to $\beta$. i.e. $\beta=\beta f_{a}(\mathrm{o}(\mathrm{a}, \alpha))$.

End for.

Step 8: $\quad$ Return $\beta$.

\subsection{Four Stage Decryption Algorithm}

Let $\mathrm{A}$ be the input alphabet, a finite ordered set of symbols comprising the language. Let $\mathrm{K}=\mathrm{K}_{0} \quad \mathrm{~K}_{1} \quad \mathrm{~K}_{2}$ be the three stage key, where each $\mathrm{Ki}$ is an element of $\mathrm{A}+$, the set of nonnull strings of A. Let $\alpha \in A+$ be the string to be encrypted. The following steps encrypt $\beta$ into $\alpha$.[12]

Step 1: Construct $Z=\cup_{a \in A} a A^{\text {index }\left(k_{0}(\text { index }(a))\right.}$ and let $\mathrm{m}$ $=|Z|=|\mathrm{A}|\left(\sum a \in \mathrm{A}\right.$ index $\left(\mathrm{K}_{0}(\operatorname{index}(\mathrm{a}))\right)$, where $\mathrm{K}_{0}(\mathrm{i})$ stands for the $\left(\mathrm{i} \bmod \left|\mathrm{K}_{0}\right|\right)^{\text {th }}+1$ letter of $\mathrm{K}_{0}$.

Step 2: Construct a permutation matrix $M$ of order $m$ from $K_{1}$ as follows: Let $\mathrm{j}=1$ and $\mathrm{p}=1$.

While $\mathrm{j}<=\mathrm{m}$ do

Let $\mathrm{i}$ be the index of $\mathrm{p}^{\text {th }}$ letter of $\mathrm{K}_{1}$.

If $i$ is a repetition, increment until an unrepeated number is obtained.

Let $\delta_{\mathrm{i}}$ be a column vector of size $\mathrm{m}$ with $\mathrm{i}^{\text {th }}$ entry as a 1 and all other entries as 0 .

Let $\delta_{i}$ be the $\mathrm{j}^{\text {th }}$ column of the matrix M.
Increment $\mathrm{j}$.

Let $\mathrm{p}=\left(\mathrm{j} \bmod \left|\mathrm{K}_{1}\right|\right)+1$.

End while.

Step 3: Let $Y=Z M$. ( $Y$ is a permutation of $Z$ ).

Step 4: Construct an n-partition of $m$ using $K_{2}$ as follows: Let $\mathrm{m}_{1}, \mathrm{~m}_{2}, \ldots, \mathrm{m}_{\mathrm{n}}$ be the indices of the letters of the word $\mathrm{K}_{2}$ taken in round robin pattern. Letq $=\mathrm{m}_{1}+\mathrm{m}_{2}+\cdots+\mathrm{m}_{\mathrm{n}}$ and $r=|Z|$ div $q$ (quotient of $|Z|$ divided by $q$ ). Partition $Y$ by taking in order the r.m $m_{1}, r . m_{2}, \ldots, r . m_{n-1}$ the remaing elements as the last block. Let these blocks be $\mathrm{Y}_{1}, \mathrm{Y}_{2}, \ldots, \mathrm{Y}_{\mathrm{n}}$.

Step 5: Let $\alpha$ be a null string.

Step 6: Let $a$ be the first letter of $\beta$ (by letter we mean an element of A).

Step7: Compute $\mathrm{q}=$ index $\left(\mathrm{K}_{0}(\right.$ index $(a))$.

Step8: Consider a prestring of length $\mathrm{q}$ of $\beta$. Let this prestring be $\gamma$.

Step9: Search $\gamma$ in the blocks of partitioned output alphabet. Suppose $\gamma$ belongs to the block $\mathrm{Y}_{\mathrm{k}}$. Let $a_{\mathrm{k}}$ be the input alphabet corresponding to the block $\mathrm{Y}_{\mathrm{k}}$.

Step10: Let $a_{\mathrm{k}}$ to be concatenated to $\alpha$.

Step11: Delete $\gamma$ from $\beta$.

Step12: Repeat the step 6 through 11 taking $\beta$ as the resulting string obtained in step 11 until $\beta$ becomes null.

Step13: Return $\alpha$.

\section{GENERATED PARTITIONED OUTPUT CRYPTO SYSTEM}

Generated Partitioned Output Crypto System is a generalization of the Four Stage Encryption System [2]. It reveals the central idea behind the four stage encryption System and allows the users and researchers to design the output alphabet generation as well as further encryption in their own way. The GPOCS is defined as follows.

\subsection{Definition}

A Generated Partitioned Output Crypto System (GPOCS) is a sextuple $\left(\mathrm{A}, \mathrm{S}, \mathrm{K}_{0}, \mathrm{P}, \chi, \delta\right)$ where

$\mathrm{A}=\left\{\mathrm{a}_{1}, \mathrm{a}_{2}, \ldots, \mathrm{a}_{\mathrm{n}}\right\}$ is a finite set, called the set of input alphabet.

$\mathrm{S}=\left\{\mathrm{z}_{1}, \mathrm{z}_{2}, \ldots, \mathrm{z}_{\mathrm{m}}\right\}$ is a finite set $\mathrm{n} \leq \mathrm{m}$, called output generating set.

$\mathrm{K}_{0}: \mathrm{S} \rightarrow \mathrm{N}$, the set of positive integers, called output generating function.

$\mathrm{P}=\left\{\mathrm{Z}_{1}, \mathrm{Z}_{2}, \ldots, \mathrm{Z}_{\mathrm{n}}\right\}$ a partition of $\mathrm{Z}$, called key partition, where $\mathrm{Z}=\mathrm{U}_{\mathrm{a} \in \mathrm{s}}$ a $\mathrm{S}^{\mathrm{K}_{0}(\mathrm{a})-1}$

$\chi=\left(\chi_{1}, \chi_{2}, \ldots, \chi_{\mathrm{n}}\right)$ is an array of mappings;

for each $\mathrm{i}, \chi_{\mathrm{i}}: \mathrm{N} \rightarrow \mathrm{Z}_{\mathrm{i}}$, called the encrypting function array.

$\delta: \mathrm{Z} \rightarrow$ A defined by $\delta(\mathrm{z})=\mathrm{a}_{\mathrm{j}}$ if $\mathrm{z}$ belongs to $\mathrm{Z}_{\mathrm{j}}$ for any $\mathrm{z}$ in $\mathrm{Z}$, called decryption function.

Clearly a Four Stage Encryption System is a GPOCS. 


\subsection{Examples}

$\mathrm{K}_{0}, \mathrm{P}, \chi$ can be chosen by the user so that they form a key for the system. Though it looks abstract, the user can design a method to get them from ordinary style of keys usually adopted in cryptography. The partitioning of the output alphabet is done by means of a permutation followed by an npartition of $m$, where $n$ and $m$ are the sizes of the input and output alphabet respectively. Examine the following illustrations of GPOCS

\subsubsection{Example}

Let $\mathrm{K}_{0}: \mathrm{A} \rightarrow \mathbf{N}$ be defined by $\mathrm{K}_{0}(\mathrm{a})=1, \mathrm{~K}_{0}(\mathrm{~b})=2, \mathrm{~K}_{0}(\mathrm{c})=3$, $\mathrm{K}_{0}(\mathrm{~d})=2$. Then $a A^{\mathrm{K}_{0}(\mathrm{a})-1}=\{\mathrm{a}\}, \mathrm{b} \mathrm{A}^{\mathrm{K}_{0}(\mathrm{~b})-1}=\{\mathrm{ba}, \mathrm{bb}, \mathrm{bc}$, bd $\}, c^{\mathrm{K}_{0}(\mathrm{C})-1}=\{\mathrm{caa}, \mathrm{cab}, \mathrm{cac}, \mathrm{cad}, \mathrm{cba}, \mathrm{cbb}, \mathrm{cbc}, \mathrm{cbd}, \mathrm{cca}$, ccb, ccc, ccd, cda, cdb, cdc, cdd $\}$, and $\mathrm{dA}^{\mathrm{K}_{0}(\mathrm{~d})-1}=\{\mathrm{da}, \mathrm{db}$, $d c$, dd $\}$. Then $Z=\{a, b a, b b, b c, b d$, caa, cab, cac, cad, cba, cbb, cbc, cbd, cca, ccb, ccc, ccd, cda, cdb, cdc, cdd, da, db, dc, dd . Let $\mathrm{K}_{1}=(5,19,8,21,3,12,16,1,20,6,18,7,2,14,4$, $9,10,13,15,17,11)$ be the permutation matrix where ' $h$ ' represents a column containing 1 in $\mathrm{h}^{\text {th }}$ place and 0 in all other places. Then $K_{1} Z=\{b d, d b$, cac, dd, bb, cbc, ccc, a, dc, caa, da, cab, ba, cca, bc, cad, cba, cbd, ccb, ccd, cbb $\}$. Let $\mathrm{K}_{2}=(3$, 9, 5, 4), so that $P=K_{2} K_{1} Z=\{\{b d, d b, c a c\},\{d d, b b, c b c$, ccc, a, dc, caa, da, cab $\},\{b a, c c a, b c, c a d, c b a\},\{c b d, c c b$, $\mathrm{ccd}, \mathrm{cbb}\}$ \}.

Let $\chi_{1}, \chi_{2}, \chi_{3}, \chi_{4}$ be functions of periodicity 9 defined by:

TABLE I . $\square_{1}, \square_{2}, \square_{3}$, and $\square_{4}$ functions of periodicity 9.

\begin{tabular}{|l|l|l|l|l|l|l|l|l|l|}
\hline & 1 & 2 & 3 & 4 & 5 & 6 & 7 & 8 & 9 \\
\hline$\chi_{1}$ & $\mathrm{bd}$ & $\mathrm{db}$ & $\mathrm{cac}$ & $\mathrm{db}$ & $\mathrm{bd}$ & $\mathrm{db}$ & $\mathrm{cac}$ & $\mathrm{db}$ & $\mathrm{bd}$ \\
\hline$\chi_{2}$ & $\mathrm{cbc}$ & $\mathrm{dc}$ & $\mathrm{cab}$ & $\mathrm{dd}$ & $\mathrm{ccc}$ & $\mathrm{caa}$ & $\mathrm{a}$ & $\mathrm{da}$ & $\mathrm{bb}$ \\
\hline$\chi_{3}$ & $\mathrm{cca}$ & $\mathrm{cba}$ & $\mathrm{bc}$ & $\mathrm{ba}$ & $\mathrm{cad}$ & $\mathrm{cba}$ & $\mathrm{cca}$ & $\mathrm{ba}$ & $\mathrm{cad}$ \\
\hline$\chi_{4}$ & $\mathrm{cbb}$ & $\mathrm{cbd}$ & $\mathrm{ccd}$ & $\mathrm{ccb}$ & $\mathrm{cbd}$ & $\mathrm{cbb}$ & $\mathrm{ccb}$ & $\mathrm{ccd}$ & $\mathrm{cbb}$ \\
\hline
\end{tabular}

Taking $\chi$ as $\left\{\chi_{1}, \chi_{2}, \chi_{3}, \chi_{4}\right\}$.

Define $\delta: \mathrm{Z} \rightarrow$ A by $\delta(\mathrm{z}) \quad=\mathrm{a}$, if $\mathrm{z} \in\{\mathrm{bd}, \mathrm{db}, \mathrm{cac}\}$

$=\mathrm{b}$, if $\mathrm{z} \in\{\mathrm{dd}, \mathrm{bb}, \mathrm{cbc}, \mathrm{ccc}, \mathrm{a}, \mathrm{dc}, \mathrm{caa}, \mathrm{da}, \mathrm{cab}\}$

$=\mathrm{c}$, if $\mathrm{z} \in\{\mathrm{ba}, \mathrm{cca}, \mathrm{bc}, \mathrm{cad}, \mathrm{cba}\}$

$=\mathrm{d}$, if $\mathrm{z} \in\{\mathrm{cbd}, \mathrm{ccb}, \mathrm{ccd}, \mathrm{cbb}\}$.

Then $\left(\mathrm{A}, \mathrm{S}, \mathrm{K}_{0}, \mathrm{P}, \chi, \delta\right)$ is a GPOCS.

With this GPOCS, the plain text 'baddadcab' encrypts as 'cbcbdcbbcbddbccdccacacdc'. To decrypt, we use $\mathrm{K}_{0}, \mathrm{~K}_{1}$, and $\mathrm{K}_{2}$ to find the partitioned output. The first character in the cipher text is ' $c$ ' and $\mathrm{K}_{0}(\mathrm{c})=3$. So we consider the first three characters, which can be decrypted as ' $b$ '. The next character is ' $\mathrm{b}$ ' and $\mathrm{K}_{0}(\mathrm{~b})=2$. So the next two characters are decrypted as ' $a$ '. Proceeding in this way, the cipher text gets decrypted into 'baddadcab'.

This example is almost a Four Stage Encryption System, except that $\mathrm{K}_{0}, \mathrm{~K}_{1}, \mathrm{~K}_{2}, \mathrm{~K}_{3}$ are designed from a string of input alphabet.

\subsubsection{Example}

Let $\mathrm{A}=\{\mathrm{g}, \mathrm{h}, \mathrm{i}, \mathrm{k}\}$.

Let $\mathrm{S}=\{1, \mathrm{~m}, \mathrm{n}, \mathrm{o}, \mathrm{p}, \mathrm{q}\}$ is output alphabet generating function. Let $\mathrm{K}_{0}: \mathrm{S} \rightarrow \mathbf{N}$ be defined by $\mathrm{K}_{0}(\mathrm{l})=3, \mathrm{~K}_{0}(\mathrm{~m})=2$, $\mathrm{K}_{0}(\mathrm{n})=3, \mathrm{~K}_{0}(\mathrm{o})=2, \mathrm{~K}_{0}(\mathrm{p})=3, \mathrm{~K}_{0}(\mathrm{q})=2$.
The output alphabet $Z$ contains 126 elements, each of which is a string of input alphabet. Let $\mathrm{K}_{1}=\{120,125,123,122,121$, $126,110,111,112,113,117,119,118,114,115,116,109$, $108,107,105,104,1,2,103,101,106,100,62,63,64,60$, $43,44,46,45,47,49,40,41,42,48,59,58,57,53,52$, $51,56,55,54,124,30,31,34,37,35,36,38,39,33,32$, $90,94,95,97,98,99,92,93,91,96,70,71,74,75,77,78$, $79,72,73,76,1,9,8,7,6,4,3,2,5,10,22,21,24,25,27$, $28,26,23,29,20,11,14,15,17,16,18,19,12,13,88,80$, $89,87,84,83,81,85,86,82,69,65,66,61,67,68,50\}$ be a permutation matrix where $h$ represents a column containing 1 in $h^{\text {th }}$ place and 0 in all other places. Then $\mathrm{K}_{1} \mathrm{Z}=\{\mathrm{pqq}, \mathrm{qp}$, $\mathrm{qn}, \ldots, \mathrm{nmm}\}$ is the permuted output alphabet.

Let $\mathrm{K}_{2}=(25,30,40,31)$, so that $\mathrm{P}=\mathrm{K}_{2} \mathrm{~K}_{1} \mathrm{Z}=\{\{$ pqq, qp qn, qm, ql, qq, ppm, ppn, ppo, ppp, pqn, pqp, pqo, ppq, pql, pqm, ppl, poq, pop, pon, pom, pnq, pol, pnp, poo $\}$, \{pno, nom, non, noo, nnq, nll, nlm, nlo, nln, nlp, nml, mo, mp, mq, nlq, nnp, nno, nnn, nmp, nmo, nmn, nnm, nnl, nmq, qo, lpq, lql, lqo, ml, lqp $\},\{l q q, \mathrm{~mm}, \mathrm{mn}, \mathrm{lqn}, \mathrm{lqm}, \mathrm{plq}, \mathrm{pmo}, \mathrm{pmp}, \mathrm{pnl}$, pnm, pnn, pmm, pmn, pml, pmq, npo, npp, nqm, nqn, nqp, nqq, ol, npq, nql, nqo, 11l, lmn, lmm, lml, llq, llo, lln, llm, llp, lmo, loo, lon, loq, lpl, lpn \}, \{lpo, lpm, lop, lpp, lom, lmp, lnm, lnn, lnp, lno, lnq, lol, lnq, lnl, plo, om, plp, pln, oq, op, on, pll, plm, oo, npn, nop, noq, nol, npl, npm, nmm \}\}.

Let $\chi=\left\{\chi_{1}, \chi_{2}, \quad \chi_{3}, \quad \chi_{4}\right\}$ be defined by: $\chi_{1}(\mathrm{n})=((3 \mathrm{n}+5)$ $\bmod 25)+1, \chi_{2}(\mathrm{n})=((\mathrm{n}+3) \bmod 30)+1, \chi_{3}(\mathrm{n})=((\mathrm{n}+7)$ $\bmod 40)+1, \chi_{4}(\mathrm{n})=((\mathrm{n}+3) \bmod 31)+1$. Let $\delta$ be defined as in the definition. Clearly $\left(\mathrm{A}, \mathrm{S}, \mathrm{K}_{0}, \mathrm{P}, \chi, \delta\right)$ is a GPOCS.

The encrypted text of the plaintext 'ghikghigh' is 'pponnopmolnmpqp'. To decrypt, $\mathrm{K}_{0}, \mathrm{~K}_{1}$, and $\mathrm{K}_{2}$ are used to find the partitioned output. The first character in the cipher text is ' $\mathrm{p}$ ' and $\mathrm{K}_{0}(\mathrm{p})=3$. So we consider the first three characters, which can be decrypted as ' $\mathrm{g}$ '. The next character is ' $n$ ' and $\mathrm{K}_{0}(\mathrm{n})=3$. So the next three characters are decrypted as ' $h$ '. Proceeding in this way, the cipher text gets decrypted into 'ghikghigh'.

\subsubsection{Example}

Let $\mathrm{A}=\{\mathrm{a}, \mathrm{b}, \mathrm{c}, \mathrm{d}\}$.

Let $S=\{\mathrm{s}, \mathrm{t}, \mathrm{u}, \mathrm{v}, \mathrm{w}\}$ is output alphabet generating function.

Let $\mathrm{K}_{0}: \mathrm{S} \rightarrow \mathbf{N}$ be defined by $\mathrm{K}_{0}(\mathrm{~s})=4, \mathrm{~K}_{0}(\mathrm{t})=2, \mathrm{~K}_{0}(\mathrm{u})=3$, $\mathrm{K}_{0}(\mathrm{v})=2 . \mathrm{K}_{0}(\mathrm{w})=3$. The output alphabet $\mathrm{Z}$ contains 185 elements. A permutation matrix $K_{1}$ of order 185 is taken and $\mathrm{K}_{1} \mathrm{Z}$ is computed. Let $\mathrm{K}_{2}=(30,45,76,34)$ and compute $\mathrm{P}=$ $\mathrm{K}_{2} \mathrm{~K}_{1} \mathrm{Z}$. Let $\chi=\left\{\chi_{1}, \chi_{2}, \chi_{3}, \chi_{4}\right\}$ be random functions from $\mathrm{N}$ into the respective blocks and $\delta$ be defined as in the definition. Clearly $\left(\mathrm{A}, \mathrm{S}, \mathrm{K}_{0}, \mathrm{P}, \chi, \delta\right)$ is a GPOCS.

With suitable choice of $\mathrm{K}_{2}$ and $\chi$, the plain text 'cadbaddad' is encrypted into 'stsvwwsvuwusswuvu vuvtstu tuuwuvuvu'.

\subsection{A Way to design Keys in GPOCS}

Given an output generating set $\mathrm{S}$, a key $\mathrm{K}_{0}$ is defined as a function from the set $S$ in to the set of natural numbers N. For each element $a \in S, S^{K_{0}(a)-1}$ is computed. This set is prefixed with the element ' $a$ '. In other words, the set $\mathrm{aS}^{\mathrm{K}_{0}}(\mathrm{a})-1$ is computed for each element $\mathrm{a} \in \mathrm{S}$. Now the output alphabet is the union of all such sets. Sothe output alphabet $\mathrm{Z}=\mathrm{U}_{\mathrm{a} \in \mathrm{s}}$ a $\mathrm{S}^{\mathrm{K}_{0}(\mathrm{a})-1}$. The prefixing with the element ' $\mathrm{a}$ ' is done to ensure that no element in the output alphabet is a prefix of any other element. Consider an $\mathrm{m} \times \mathrm{m}$ permutation matrix $\mathrm{K}_{1}$ (a binary matrix with exactly one 1 each row and each column), where $m$ is the number of elements in the output alphabet. This matrix $\mathrm{K}_{1}$ serves as second stage key. $\mathrm{K}_{1}$ 
$\mathrm{Z}, \mathrm{Z}$ treated as column matrix, permutes the elements of $\mathrm{Z}$. Let this permutation be $U=K_{1} Z=\left(u_{1}, u_{2}, \ldots, u_{m}\right)$. The third stage key $K_{2}=\left(m_{1}, m_{2}, \ldots, m_{n}\right)$ is an n-partition of $m$ (an ordered $n$-tuple of positive integers whose sum is $m$ ). Then the partition $P=\left\{Z_{1}, Z_{2}, \ldots, Z_{n}\right\}$ is given by $Z_{1}=\left\{u_{1}, \ldots\right.$, $\left.\mathrm{u}_{\mathrm{m} 1}\right\}$, the set of first $\mathrm{m}_{1}$ elements of $\mathrm{U}, \mathrm{Z}_{2}=\left\{\mathrm{u}_{\mathrm{m} 1+1}, \ldots\right.$, $\left.u_{m 1+m}\right\}$, the set of all next $m_{2}$ elements of $U$ and so on. The fourth stage key is a set of $n$ functions $\chi=\left\{\chi_{1}, \chi_{2}, \ldots \chi_{n}\right\}$ where $\chi_{\mathrm{i}}: \mathrm{N} \rightarrow \mathrm{Z}_{\mathrm{i}}$ for each $\mathrm{i}$. Here these may be any functions of our choice. We may consider a function with $\mathrm{N}_{\mathrm{p}}=\{1,2, \ldots$ $, \mathrm{p}\}$ as the domain or a periodic function of periodicity $\mathrm{p}$ for a sufficiently large p. Again in any of these cases these $n$ functions can be associated with $\mathrm{n}$ binary matrices. If $\mathrm{N}_{\mathrm{p}}$ is considered as the domain, for each $i$, let $B_{i}$ be $a m_{i} \times p$ binary matrix in which each row contains exactly one 1 . Now $\chi_{\mathrm{i}}(\mathrm{n})=$ $Z_{i} B_{i} e_{n}$ in which $e_{n}=(0,0, \ldots, 1, \ldots, 0) 1$ being at the $n^{\text {th }}$ place. In the later case also, $\chi_{i}(n)=Z_{i} B_{i} e_{n}(\bmod p)+1$. The third stage key may be defined as $K_{3}=\left\{B_{i}: 1 \leq i \leq n\right\}$. So, finally we have $\mathrm{K}_{0}, \mathrm{~K}_{1}, \mathrm{~K}_{2}, \mathrm{~K}_{3}$ the four stages of keys for encryption. We illustrate the above key structure by an example.

\subsubsection{Example}

Let $\mathrm{A}=\{\mathrm{e}, \mathrm{f}, \mathrm{g}, \mathrm{h}\}, \mathrm{S}=\{\mathrm{a}, \mathrm{b}, \mathrm{c}, \mathrm{d}\}$, and $\mathrm{K}_{0}: \mathrm{S} \rightarrow \mathrm{N}$ be defined as $\mathrm{K}_{0}(\mathrm{a})=2, \mathrm{~K}_{0}(\mathrm{~b})=2, \mathrm{~K}_{0}(\mathrm{c})=2, \mathrm{~K}_{0}(\mathrm{~d})=2$. Then the output alphabet $Z=\{a a, a b, a c, a d, b a, b b, b c, b d, c a, c b$, $c c, c d, d a, d b, d c, d d\}$. Let $K_{1}, K_{2}$ be defined as follows:

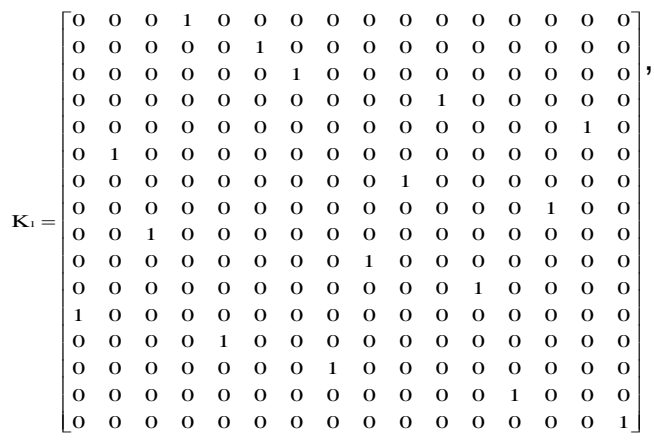

$\mathrm{K}_{2}=(5,3,3,5)$.

Then $K_{1} Z=\{a d, b b, b c, c c, d c, a b, c b, d b, a c, c a, c d, a a, b a$, $\mathrm{bd}, \mathrm{da}, \mathrm{dd}\}$ and $\mathrm{P}=\{\{\mathrm{ad}, \mathrm{bb}, \mathrm{bc}, \mathrm{cc}, \mathrm{dc}\},\{\mathrm{ab}, \mathrm{cb}, \mathrm{db}\},\{\mathrm{ac}, \mathrm{ca}$, cd $\},\{a a, b a, b d, d a, d d\}$.

Let $\mathrm{p}=8$ and the functions be defined as periodic functions by the following matrices:

$$
\begin{aligned}
\mathrm{B}_{1} & =\left[\begin{array}{llllllll}
1 & 0 & 0 & 0 & 0 & 0 & 0 & 0 \\
0 & 1 & 0 & 0 & 0 & 0 & 0 & 1 \\
0 & 0 & 1 & 0 & 0 & 0 & 1 & 0 \\
0 & 0 & 0 & 1 & 0 & 1 & 0 & 0 \\
0 & 0 & 0 & 0 & 1 & 0 & 0 & 0
\end{array}\right], \\
\mathrm{B}_{2} & =\left[\begin{array}{llllllll}
1 & 0 & 1 & 0 & 0 & 1 & 0 & 0 \\
0 & 1 & 0 & 0 & 1 & 0 & 1 & 0 \\
0 & 0 & 1 & 1 & 1 & 0 & 0 & 1
\end{array}\right], \\
\mathrm{B}_{3} & =\left[\begin{array}{llllllll}
1 & 0 & 1 & 0 & 1 & 0 & 0 & 1 \\
0 & 1 & 0 & 1 & 0 & 0 & 1 & 0 \\
0 & 0 & 1 & 0 & 0 & 1 & 0 & 0
\end{array}\right], \\
\mathrm{B}_{4} & =\left[\begin{array}{llllllll}
1 & 0 & 0 & 0 & 0 & 0 & 0 & 0 \\
0 & 1 & 0 & 0 & 0 & 0 & 0 & 1 \\
0 & 0 & 1 & 0 & 0 & 0 & 1 & 0 \\
0 & 0 & 0 & 1 & 0 & 1 & 0 & 0 \\
0 & 0 & 0 & 0 & 1 & 0 & 0 & 0
\end{array}\right]
\end{aligned}
$$

Clearly, $\chi=\left\{\chi_{1}, \chi_{2}, \chi_{3}, \chi_{4}\right\}$ where

$\chi_{1}: 1 \rightarrow \mathrm{ad}, 2 \rightarrow \mathrm{bb}, 3 \rightarrow \mathrm{bc}, 4 \rightarrow \mathrm{cc}, 5 \rightarrow \mathrm{dc}, 6 \rightarrow \mathrm{cc}, 7 \rightarrow$ $\mathrm{bc}, 8 \rightarrow \mathrm{bb}$

$$
\begin{aligned}
& \chi_{2}: 1 \rightarrow \mathrm{ab}, 2 \rightarrow \mathrm{cb}, 3 \rightarrow \mathrm{db}, 4 \rightarrow \mathrm{db}, 5 \rightarrow \mathrm{cb}, 6 \rightarrow \mathrm{ab}, 7 \rightarrow \\
& \mathrm{cb}, 8 \rightarrow \mathrm{db} \\
& \chi_{3}: 1 \rightarrow \mathrm{ac}, 2 \rightarrow \mathrm{ca}, 3 \rightarrow \mathrm{cd}, 4 \rightarrow \mathrm{ca}, 5 \rightarrow \mathrm{ac}, 6 \rightarrow \mathrm{cd}, 7 \rightarrow \\
& \mathrm{ca}, 8 \rightarrow \mathrm{ac} \\
& \chi_{4}: 1 \rightarrow \mathrm{aa}, 2 \rightarrow \mathrm{ba}, 3 \rightarrow \mathrm{bd}, 4 \rightarrow \mathrm{da}, 5 \rightarrow \mathrm{dd}, 6 \rightarrow \mathrm{ba}, 7 \rightarrow \\
& \mathrm{bd}, 8 \rightarrow \mathrm{da} \\
& \text { and } \chi_{\mathrm{i}}(\mathrm{n})=\chi_{\mathrm{i}}((\mathrm{n} \% 8)+1) \text { for } \mathrm{n}>8 \text { for any } 1 \leq \mathrm{i} \leq 4 .
\end{aligned}
$$

\subsection{An application of GPOCS}

In the real world requirement, we need to have the keys as words, numbers, or some other pattern. The users cannot remember or understand the actual process hidden from the users. Here, as a first application, a sentence of four words, each word being sufficiently long is considered as a key. From these words, we build the four stages of keys, which are functions with appropriate domains and ranges. The following example illustrates the procedure

\subsubsection{Example}

Let the input alphabet $\mathrm{A}=\{\mathrm{a}, \mathrm{b}, \mathrm{c}, \mathrm{d}\}$.Let the key be 'bad acad acb dacab'.

From the first word 'bad', the key $\mathrm{K}_{0}$ is: $\mathrm{K}_{0}(\mathrm{a})=2$, the index of the first letter of the word 'bad' in $\mathrm{A} . \mathrm{K}_{0}(\mathrm{~b})=1$, the index of the second letter of the word 'bad' in $\mathrm{A} . \mathrm{K}_{0}(\mathrm{c})=4$, the index of the third letter of the word 'bad' in $A . K_{0}(d)=2$, the index of the first letter of the word 'bad', since all the letters of the key are exhausted. In general, $\mathrm{K}_{0}\left(\mathrm{a}_{\mathrm{i}}\right)=\mathrm{j}$, where $\mathrm{a}_{\mathrm{i}}$ is the $\mathrm{i}^{\text {th }}$ letter of the first word in the key.

The output alphabet is $Z=\{a a, a b, a c, a d, b$, caaa, caab, caac, caad, caba, cabb, cabc, cabd, caca, cacb, cacc, cacd, cada, cadb, cadc, cadd, cbaa, cbab, cbac, cbad, cbba, cbbb, cbbc, cbbd, cbca, cbcb, cbcc, cbcd, cbda, cbdb, cbdc, cbdd, ccaa, ccab, ccac, ccad, ccba, ccbb, ccbc, ccbd, ccca, cccb, cccc, cccd, ccda, ccdb, ccdc, ccdd, cdaa, cdab, cdac, cdad, cdba, cdbb, cdbc, cdbd, cdca, cdcb, cdcc, cdcd, cdda, cddb, cddc, cddd, da, db, dc, dd $\}$. Clearly, $|\mathrm{Z}|=73$.

Let us consider the indices of the letters of the second word in order. If some number repeats, replace it by some other number within the length of the word. For example, increment the number until a number that is different from the existing ones is arrived. Let consider a matrix with the columns corresponding to these numbers. i.e., for the number $\mathrm{k}$, the corresponding column contains 1 in its $\mathrm{k}^{\text {th }}$ place and $0 \mathrm{~s}$ in the rest of the places. These vectors are repeated cyclically incrementing the positions of $1 \mathrm{~s}$ until we get $|\mathrm{Z}|$ number of vectors.

Coming to the present example, the second word of the key is 'acad'. The corresponding numbers are 1,3,1, 4 respectively. 1 is repeated and hence the second one is replaced by 3 . So the numbers are $1,3,2,4$. Now the matrix representing the key $K_{1}$ is $(1,3,2,4,5,7,6,8,9,11,10,12, \ldots \ldots, 73)$. Now, $\mathrm{ZK}_{1}=\{\mathrm{aa}, \mathrm{ac}, \mathrm{ab}, \mathrm{ad}, \mathrm{b}, \mathrm{caab}, \mathrm{caaa}, \mathrm{caac}, \mathrm{caad}, \mathrm{cabb}, \mathrm{caba}$, cabc, cabd, cacb, caca, cacc, cacd, cadb, cada, cadc, cadd, cbab, cbaa, cbac, cbad, cbbb, cbba, cbbc, cbbd, cbcb, cbca, cbcc, cbcd, cbdb, cbda, cbdc, cbdd, ccab, ccaa, ccac, ccad, ccbb, ccba, ccbc, ccbd, cccb, ccca, cccc, cccd, ccdb, ccda, ccdc, ccdd, cdab, cdaa, cdac, cdad, cdbb, cdba, cdbc, cdbd, cdcb, cdca, cdcc, cdcd, cddb, cdda, cddc, cddd, db, da, dc, dd $\}$.

Now it is turn of the third word. The indices $i_{1}, i_{2}, \ldots, i_{k}$ (generally $\mathrm{k}$, the length of the third word is less than the number of input alphabets) of the letters of the word are taken. 
The numbers are extended to the size $\mathrm{n}$ of the input alphabet by repeating these numbers cyclically getting $i_{1}, i_{2}, \ldots, i_{n}$. Let their total be $\mathrm{t}=\mathrm{i}_{1}+\mathrm{i}_{2}+\ldots+\mathrm{i}_{\mathrm{n}}$. Let $\mathrm{q}$ be the quotient obtained by dividing $|\mathrm{Z}|$ by $\mathrm{t}$. Let the sizes of the blocks be q. $i_{1}, q . i_{2}, \ldots, q . i_{k-1}$, and $t-\left(q . i_{1}+q . i_{2}+\ldots, q . i_{k-1}\right)$. i.e., the key $\mathrm{K}_{2}=\left(\mathrm{q} \cdot \mathrm{i}_{1}, \mathrm{q} \cdot \mathrm{i}_{2}, \ldots, \mathrm{q} \cdot \mathrm{i}_{\mathrm{k}-1}, \mathrm{t}-\left(\mathrm{q} \cdot \mathrm{i}_{1}+\mathrm{q} \cdot \mathrm{i}_{2}+\ldots, \mathrm{q} \cdot \mathrm{i}_{\mathrm{k}-1}\right)\right) \cdot \mathrm{ZK}_{1}$ is now partitioned according to these block sizes to get $\mathrm{P}$, the key partition.

In our example, the third word is 'acb'. The indices are 1, 3, 2. Extending, we get $1,3,2,1$. The sum $t=1+3+2+1=7$. The quotient $\mathrm{q}=10$, when 73 is divided by 7 . A partition of 73 is $(10,30,20,13)$. Then $P=\{\{a a, a c, a b, a d, b, c a a b$, caaa, caac, caad, cabb\}, \{caba, cabc, cabd, cacb, caca, cacc, cacd, cadb, cada, cadc, cadd, cbab, cbaa, cbac, cbad, cbbb, cbba, cbbc, cbbd, cbcb, cbca, cbcc, cbcd, cbdb, cbda, cbdc, cbdd, ccab, ccaa, ccac $\},\{c c a d, c c b b, c c b a, c c b c, c c b d, c c c b$, ccca, cccc, cccd, ccdb, ccda, ccde, ccdd, cdab, cdaa, cdac, cdad, cdbb, cdba, cdbc $\}$, \{cdbd, cdcb, cdca, cdcc, cdcd, cddb, cdda, cddc, cddd, db, da, dc, dd \} \}.

Finally, the fourth word 'dacabc' generates the encryption function key array as follows. The indices of the word are 4, $1,3,1-$ a repetition and replaced by 2 ( 1 is incremented until an unrepeated number is arrived at), index of the next letter is 2 - again a repetition and is replaced by 5 , and finally, the index of the letter is 3 , which is replaced by 6 . So we obtained a sequence of numbers: 4, 1, 3, 2, 5, 6. We can extend this sequence by adding 6 , the length of the word to these numbers and the 12,18 , the multiples of 6 . The sequence obtained is: 4 , $1,3,2,5,6,10,7,9,8,11,12,16,13,15,14,17,18 \ldots$ From this sequence, the first 30 (the maximum of $10,30,20$, and 13) numbers are taken to form the matrix $B_{1}$ and next 30 to form $B_{2}$ and so on. For example, $B 1=\left(e_{4} e_{1} e_{3} e_{2} e_{5} e_{6} e_{10} e_{7}\right.$ $\left.e_{9} e_{8} \ldots\right)$, a $10 \times 30$ matrix, where $e_{i}$ stands for a column matrix of size 10 with 1 in $((\mathrm{i} \bmod 10)+1)^{\text {th }}$ place and all other entries are zeros. $\mathrm{B}_{2}$ is a $30 \times 30, \mathrm{~B}_{3}$ is a $20 \times 30$, and $\mathrm{B}_{4}$ is a $13 \times 30$ matrices obtained in a similar manner. These matrices form the key $\mathrm{K}_{3}=\left(\mathrm{B}_{1}, \mathrm{~B}_{2}, \mathrm{~B}_{3}, \mathrm{~B}_{4}\right)$. Now, given any plain text we can encode it using this key structure $\left(\mathrm{K}_{0}\right.$, $\mathrm{K}_{1}, \mathrm{~K}_{2}, \mathrm{~K}_{3}$ ) and any cipher text encoded in this way can be decoded using $\left(\mathrm{K}_{0}, \mathrm{~K}_{1}, \mathrm{~K}_{2}, \mathrm{~K}_{3}\right)$.

\section{PARTITIONED OUTPUT CRYPTO SYSTEM}

To further study the properties of the system, a pure mathematical system, called Partitioned Output Cryptosystem, is proposed. A sketch of Partitioned Output Cryptosystem and its properties are presented in this section.

\subsection{Definition}

A Partitioned Output Crypto System (POCS) is a quintuple $(\mathrm{A}, \mathrm{Z}, \mathrm{P}, \chi, \delta)$ where

$\mathrm{A}=\left\{\mathrm{a}_{1}, \mathrm{a}_{2}, \ldots, \mathrm{a}_{\mathrm{n}}\right\}$ is a finite set, called the set of input alphabet

$\mathrm{Z}=\left\{\mathrm{z}_{1}, \mathrm{z}_{2}, \ldots, \mathrm{z}_{\mathrm{m}}\right\}$ is a finite set $(\mathrm{m} \geq \mathrm{n})$, called the set of output alphabet.

$\mathrm{P}=\{\mathrm{Z} 1, \mathrm{Z} 2, \ldots, \mathrm{Zn}\}$ a partition of $\mathrm{Z}$, called key partition.

$\chi=\left\{\chi_{1}, \chi_{2}, \ldots, \chi_{\mathrm{n}}\right\}$ is a set of mappings; for each $\mathrm{i}, \chi_{\mathrm{i}}: \mathrm{N} \rightarrow$ $\mathrm{Z}_{\mathrm{i}}$, called the encrypting function array.

$\delta: \mathrm{Z} \rightarrow$ A defined by $\delta(\mathrm{z})=\mathrm{a}_{\mathrm{j}}$ if $\mathrm{z}$ belongs to $\mathrm{Z}_{\mathrm{j}}$ for any $\mathrm{z}$ in $\mathrm{Z}$, called decryption function.

For any word $w=\mathrm{a}_{\mathrm{i} 1} \mathrm{a}_{\mathrm{i} 2} \ldots \mathrm{a}_{\mathrm{ik}}$ in $\mathrm{A}^{*}$, define $\chi(w)=\chi_{\mathrm{i} 1}\left(\mathrm{o}\left(\mathrm{a}_{\mathrm{i} 1}, w\right)\right)$ $\chi_{\mathrm{i} 2}\left(\mathrm{o}\left(\mathrm{a}_{\mathrm{i} 2}, w\right)\right) \ldots \chi_{\mathrm{ik}}\left(\mathrm{o}\left(\mathrm{a}_{\mathrm{ik}}, w\right)\right)$, where $\mathrm{o}(\mathrm{a}, w)$ stands for the order of occurrence of a in the word $w$. i.e., Each $\mathrm{a}_{\mathrm{j}}$ of $w$ is replaced by $\chi_{\mathrm{j}}(\mathrm{p})$, where $\mathrm{a}_{\mathrm{j}}$ is the $\mathrm{p}^{\text {th }}$ occurrence of itself in $w$. $\chi(w)$ is called the encrypted word of the word $w$. The decrypting function, $\delta$ can be extended to $Z^{*}$, as $\Delta(u)=\delta\left(\mathrm{z}_{\mathrm{j} 1}\right)$ $\delta\left(\mathrm{z}_{\mathrm{j} 2}\right) \ldots \delta\left(\mathrm{z}_{\mathrm{jk}}\right)$ for any $u=\mathrm{z}_{\mathrm{j} 1} \mathrm{z}_{\mathrm{j} 2} \ldots \mathrm{z}_{\mathrm{jk}}$ in $\mathrm{Z}^{*} . \Delta(u)$ is called the decrypted word of the word $u$. Clearly $\delta\left(\chi_{\mathrm{i}}(n)\right)=\mathrm{a}_{\mathrm{i}}$ for all $\mathrm{i}, 1 \leq \mathrm{i} \leq \mathrm{n}$; and hence $\Delta(\chi(w))=w$ for all $w$ in $\mathrm{Z}^{*}$.

\subsection{Examples}

4.2.1 Example

Let $\mathrm{A}=\{\mathrm{a}, \mathrm{b}, \mathrm{c}, \mathrm{d}\}$,

$\mathrm{Z}=\{\mathrm{e}, \mathrm{f}, \mathrm{g}, \mathrm{h}, \mathrm{j}, \mathrm{k}, \mathrm{l}, \mathrm{m}, \mathrm{n}, \mathrm{o}, \mathrm{p}, \mathrm{q}, \mathrm{r}, \mathrm{s}, \mathrm{t}, \mathrm{u}, \mathrm{v}, \mathrm{w}, \mathrm{x}, \mathrm{y}, \mathrm{z}\}$

$P=\{\{h, k, 1, p, t, x, z\},\{f, o, s, y\},\{g, n, q, v\},\{e, j, m, r, u$, w $\}$ \}

$\chi=\left\{\chi_{1}, \chi_{2}, \chi_{3}, \chi_{4}\right\}$ where $\chi_{1}: 1 \rightarrow \mathrm{t}, 2 \rightarrow \mathrm{h}, 3 \rightarrow \mathrm{x}, 4 \rightarrow 1,5 \rightarrow$ $\mathrm{k}, 6 \rightarrow \mathrm{z}, 7 \rightarrow \mathrm{p}$ and $\chi_{1}(\mathrm{n})=\chi_{1}((\mathrm{n} \% 7)+1)$ for $\mathrm{n}>7$.

$\chi_{2}: 1 \rightarrow \mathrm{o}, 2 \rightarrow \mathrm{s}, 3 \rightarrow \mathrm{y}, 4 \rightarrow \mathrm{f}$, and $\chi 2(\mathrm{n})=\chi 2((\mathrm{n} \% 4)+1)$ for $\mathrm{n}>4$.

$\chi_{3}: 1 \rightarrow \mathrm{g}, 2 \rightarrow \mathrm{q}, 3 \rightarrow \mathrm{n}, 4 \rightarrow \mathrm{v}$, and $\chi 3(\mathrm{n})=\chi 3((\mathrm{n} \% 4)+1)$ for $\mathrm{n}>4$.

$\chi_{4}: 1 \rightarrow \mathrm{u}, 2 \rightarrow \mathrm{r}, 3 \rightarrow \mathrm{e}, 4 \rightarrow \mathrm{m}, 5 \rightarrow \mathrm{j}, 6 \rightarrow \mathrm{w}$ and $\chi_{4}(\mathrm{n})=$ $\chi_{4}((\mathrm{n} \% 6)+1)$ for $\mathrm{n}>6$, where $\mathrm{n} \% \mathrm{k}$ stands for the remainder of $\mathrm{n}$ divided by $\mathrm{k}$.

Then $(\mathrm{A}, \mathrm{Z}, \mathrm{P}, \chi)$ is a POCS.

Let $w=$ cadbaddacdadba. Then $\chi(w)$ is computed as follows. $\chi_{3}(\mathrm{o}(\mathrm{c}, w))=\chi_{3}(1)=\mathrm{g}, \chi_{1}(\mathrm{o}(\mathrm{a}, w))=\chi_{1}(1)=\mathrm{t}, \chi_{4}(\mathrm{o}(\mathrm{d}, w))=$ $\chi_{4}(1)=\mathrm{u}, \chi_{2}(\mathrm{o}(\mathrm{b}, w))=\chi_{2}(1)=\mathrm{o}, \chi_{1}(\mathrm{o}(\mathrm{a}, w))=\chi_{1}(2)=\mathrm{h}$, $\chi_{4}(\mathrm{o}(\mathrm{d}, w))=\chi_{4}(2)=\mathrm{r}, \chi_{4}(\mathrm{o}(\mathrm{d}, w))=\chi_{4}(3)=\mathrm{e}, \ldots$ etc. Hence $\chi(w)=$ gtuohrexqmljsk. The word cadbaddacdadba is encrypted as gtuohrexqmljsk. Notice that though a and $\mathrm{d}$ are repeated very often in the plain text, the encrypted text wont reflect those characteristics. Generally the encrypted text can be decrypted by a third party by knowing the frequency of characters in the plain text.

\subsubsection{Example}

Let $\mathrm{A}=\{\mathrm{a}, \mathrm{b}, \mathrm{c}, \mathrm{d}\}$

$Z=\{a a, a b, a c, a d, b a, b b, b c, b d, c a, c b, c c, c d, d a, d b, d c$, dd $\}$

$P=\{\{a d, b b, b c, c c, d c\},\{a b, c b, d b\},\{a c, c a, c d\},\{a a, b a$, bd, da, dd $\}$

$\chi=\left\{\chi_{1}, \chi_{2}, \chi_{3}, \chi_{4}\right\}$ where

$\chi_{1}: 1 \rightarrow \mathrm{ad}, 2 \rightarrow \mathrm{bb}, 3 \rightarrow \mathrm{bc}, 4 \rightarrow \mathrm{cc}, 5 \rightarrow \mathrm{dc}, 6 \rightarrow \mathrm{cc}, 7 \rightarrow$ bc, $8 \rightarrow$ bb

$\chi_{2}: 1 \rightarrow \mathrm{ab}, 2 \rightarrow \mathrm{cb}, 3 \rightarrow \mathrm{db}, 4 \rightarrow \mathrm{db}, 5 \rightarrow \mathrm{cb}, 6 \rightarrow \mathrm{ab}, 7 \rightarrow$ $\mathrm{cb}, 8 \rightarrow \mathrm{db}$

$\chi_{3}: 1 \rightarrow$ ac, $2 \rightarrow$ ca, $3 \rightarrow$ cd, $4 \rightarrow$ ca, $5 \rightarrow$ ac, $6 \rightarrow$ cd $7 \rightarrow$ ca, $8 \rightarrow$ ac

$\chi_{4}: 1 \rightarrow$ aa $2 \rightarrow$ ba, $3 \rightarrow$ bd, $4 \rightarrow$ da, $5 \rightarrow$ dd, $6 \rightarrow$ ba, $7 \rightarrow$ bd, $8 \rightarrow$ da and $\chi_{\mathrm{i}}(\mathrm{n})=\chi_{\mathrm{i}}((\mathrm{n} \% 8)+1)$ for $\mathrm{n}>8$ for any $1 \leq \mathrm{i} \leq 4$.

Then $(\mathrm{A}, \mathrm{Z}, \mathrm{P}, \chi)$ is a POCS.

Let $w=$ cadbaddacdadba. Then $\chi(w)$ is computed as follows. $\chi_{3}(\mathrm{o}(\mathrm{c}, w))=\chi_{3}(1)=\mathrm{ac}, \chi_{1}(\mathrm{o}(\mathrm{a}, w))=\chi_{1}(1)=\mathrm{ad}, \chi_{4}(\mathrm{o}(\mathrm{d}, w))$ $=\chi_{4}(1)=\mathrm{aa}, \chi_{2}(\mathrm{o}(\mathrm{b}, w))=\chi_{2}(1)=\mathrm{ab}, \chi_{1}(\mathrm{o}(\mathrm{a}, w))=\chi_{1}(2)=\mathrm{bb}$, 
$\chi_{4}(\mathrm{o}(\mathrm{d}, w))=\chi_{4}(2)=\mathrm{ba}, \chi_{4}(\mathrm{o}(\mathrm{d}, w))=\chi_{4}(3)=\mathrm{bd}, \ldots$ etc. Hence $\chi(w)=$ aaacaaabbbbabdbccadaccddcbdc.

The word cadbaddacdadba is encrypted as aaacaaabbbbabdbccadaccddcbdc. Notice that the encrypted word has double length as that of the plain text and it is difficult to decrypt the text without knowing the encryption function array.

\subsubsection{Example}

Let $\mathrm{A}=\{\mathrm{a}, \mathrm{b}, \mathrm{c}, \mathrm{d}\}$

$\mathrm{Z}=\{\mathrm{ab}, \mathrm{ac}, \mathrm{ad}, \mathrm{ba}, \mathrm{bc}, \mathrm{bd}, \mathrm{ca}, \mathrm{cb}, \mathrm{cd}, \mathrm{da}, \mathrm{db}, \mathrm{dc}, \mathrm{dd}\}$

$\mathrm{P}=\{\{\mathrm{ad}, \mathrm{bc}, \mathrm{dc}, \mathrm{db}\},\{\mathrm{ab}, \mathrm{cb}\},\{\mathrm{ac}, \mathrm{ca}, \mathrm{cd}\},\{\mathrm{ba}, \mathrm{bd}, \mathrm{da}\}\}$

$\chi=\left\{\chi 1, \chi^{2}, \chi^{3}, \chi^{4}\right\}$ where $\chi 1: 1 \rightarrow \mathrm{ad}, 2 \rightarrow \mathrm{bc}, 3 \rightarrow \mathrm{dc}, 4$

$\rightarrow \mathrm{db}$, and $\chi 1(\mathrm{n})=\chi 1(\mathrm{n} \% 4+1)$ for $\mathrm{n}>4 \chi^{2}: 1 \rightarrow \mathrm{ab}, 2 \rightarrow$ $\mathrm{cb}$, and $\chi_{2}(\mathrm{n})=\chi_{2}(\mathrm{n} \% 2+1)$ for $\mathrm{n}>2 \chi_{3}: 1 \rightarrow \mathrm{ac}, 2 \rightarrow \mathrm{ca}, 3$ $\rightarrow$

cd and $\chi_{3}(\mathrm{n} \% 3+1)$ for $\mathrm{n}>3 \quad \chi_{4}: 1 \rightarrow$ ba, $2 \rightarrow$ bd, $3 \rightarrow$ da, and $\chi_{4}(\mathrm{n} \% 3+1)$ for $\mathrm{n}>3$.

Then $(\mathrm{A}, \mathrm{Z}, \mathrm{P}, \chi)$ is a POCS.

Let $w=$ cadbaddacdadba. Then $\chi(w)=$ acadbaabbcbddadcc

abadbbdac. The word cadbaddacdadba is encrypted as acadbaabbcbddadccabadbbdac.

Notice that the encrypted word has double length as that of the plain text and it is difficult to decrypt the text without knowing the encryption function array. In the previous examples, the size of the encrypted word is a multiple of the size of the original word. This allows a chance of estimating the chance of guessing the size of the original word by considering all possible divisors of the size of the encrypted word.

\subsubsection{Example}

Let $\mathrm{A}=\{\mathrm{a}, \mathrm{b}, \mathrm{c}, \mathrm{d}\}$

$\mathrm{Z}=\{\mathrm{a}, \mathrm{ba}, \mathrm{bc}, \mathrm{bd}, \mathrm{cab}, \mathrm{cad}, \mathrm{cba}, \mathrm{cbd}, \mathrm{cda}, \mathrm{cdb}, \mathrm{dabc}, \mathrm{dacb}$, dbac, dbca, dcab, dcba

$P=\{\{c a b, b d, d c b a\},\{c a d, d a b c, a, b a, c d a, d b a c\},\{d c a b$, cdb, dbca $\},\{c b a, c b d, d a c b, b c\}\}$

$\chi=\left\{\chi_{1}, \chi_{2}, \chi_{3}, \chi_{4}\right\}$ where

$\chi_{1}: 1 \rightarrow \mathrm{cab}, 2 \rightarrow \mathrm{bd}, 3 \rightarrow \mathrm{dcba}$, and $\chi_{1}(\mathrm{n})=\chi_{1}(\mathrm{n} \% 3+1)$ for $\mathrm{n}>3$

$\chi_{2}: 1 \rightarrow$ cad, $2 \rightarrow$ dabc, $3 \rightarrow$ a, $4 \rightarrow$ ba, $5 \rightarrow$ cda, $6 \rightarrow$ dbac

$\chi_{2}(\mathrm{n})=\chi_{2}(\mathrm{n} \% 6+1)$ for $\mathrm{n}>6 \chi_{3}: 1 \rightarrow$ dcab, $2 \rightarrow$ cbd, $3 \rightarrow$ $\mathrm{dbca}$, and $\chi_{3}(\mathrm{n} \% 3+1)$ for $\mathrm{n}>3$

$\chi_{4}: 1 \rightarrow$ cba, $2 \rightarrow$ cbd, $3 \rightarrow$ dacb, $4 \rightarrow$ bc, and $\chi_{4}(\mathrm{n} \% 3+1)$ for $\mathrm{n}>4$

Then $(\mathrm{A}, \mathrm{Z}, \mathrm{P}, \chi)$ is a POCS.

Let $w=$ cadbad. Then $\chi(w)=$ dcabcabcbacadbdcbd. Since each alphabet is encoded into a word of different size, decryption without knowing the key partition. Here the output alphabet (word) has a length of 1 if it starts with a, 2 if it starts with $\mathrm{b}, 3$ if it starts with $\mathrm{c}$, and 4 if it starts with d, so that there will not be any problem in decryption.

\subsection{Elementary Properties of POCS}

We study some elementary properties of POCS and show that POCS is a generalization of a cryptosystem. Both symmetric and asymmetric ciphers can be explained through POCS.

\subsubsection{Definition}

Two cryptosystems [4], [5], [7]are k-equivalent if they encrypt and decrypt in the same way for a given key k. i.e. for any given message they give raise to the same decrypted word and vice versa.

\subsubsection{Theorem: The POCS $(A, Z, P, \chi, \delta)$ is $P$ equivalent to a Crypto system [4], [5].}

Proof: Suppose $(\mathrm{A}, \mathrm{Z}, \mathrm{P}, \chi, \delta)$ is a POCS.

Consider the cryptosystem $(\mathrm{M}, \mathrm{C}, \mathrm{K}, \mathrm{e}, \mathrm{d})$

where $\mathrm{M}=\mathrm{A}^{*}, \mathrm{C}=\mathrm{Z}^{*}, \mathrm{~K}$ is the set of all n-block partitions of $\mathrm{Z}$. For the partition $\mathrm{P}$ in $\mathrm{K}, \mathrm{e}_{\mathrm{P}}$ and $\mathrm{d}_{\mathrm{P}}$ are defined as follows: $\mathrm{e}_{\mathrm{P}}(\mathrm{m})=\chi(\mathrm{m})$ for all $\mathrm{m}$ in $\mathrm{M}$ and $\mathrm{d}_{\mathrm{P}}(\mathrm{c})=\Delta(\mathrm{c})$ for all $\mathrm{c}$ in $\mathrm{C}$. Clearly $(\mathrm{M}, \mathrm{C}, \mathrm{K}, \mathrm{e}, \mathrm{d})$ is a cryptosystem and for the key $\mathrm{P}$ in $\mathrm{K}, \mathrm{e}_{\mathrm{P}}=\chi$ and $\mathrm{d}_{\mathrm{P}}=\Delta$. Hence the $(\mathrm{A}, \mathrm{Z}, \mathrm{P}, \chi, \delta)$ is $\mathrm{P}$ equivalent to $(\mathrm{M}, \mathrm{C}, \mathrm{K}, \mathrm{e}, \mathrm{d})$.

\subsection{Three Stages of Key design in POCS}

First the output needs to be partitioned. This requires:

1. A permutation of the output alphabet.

2. A an $n$-partition of the integer $m$ (i.e. $m$ should be express as the sum of $n$ positive integers: $m=k_{1}+\ldots$ $+\mathrm{k}_{\mathrm{n}}$ ).

3. The first block of the partition $P$ contains the first $\mathrm{k}_{1}$ elements; the second block of partition $\mathrm{P}$ contains $\mathrm{k} 2$ elements and so on

After getting a partition $\mathrm{P}$, a function from A to $\mathrm{P}$ may be defined. This function may be ignored if proper care is taken in the initial permutation. We just suppose that $\alpha_{1}$ is mapped onto $P_{1}, \alpha_{2}$ is mapped onto $P_{2}$ and so on.

Now we are left with defining $\chi$. So the three levels of the key generations involve:

1. Initial permutation of the output alphabet $\mathrm{Z}$.

2. Partitioning $\mathrm{m}$.

3. Defining $\chi$.

While encoding a word, we need all these three stages of key functions. But to decode a word we need to know only function $\delta$.

4.4.1 Theorem: The POCS $(A, Z, P, \chi, \delta)$ is an asynchronous crypto system.

Proof: The proof is trivial by the previous explanation.

For any $f$ in Mfn (D, E) [3], [8], [9], [10] $f$ can be extended to $\boldsymbol{f}: \mathrm{D}^{*} \rightarrow \mathrm{E}^{*}$ as $\boldsymbol{f}(w)=f\left(\mathrm{a}_{\mathrm{i} 1}\right) f\left(\mathrm{a}_{\mathrm{i} 2}\right) \ldots f\left(\mathrm{a}_{\mathrm{ik}}\right)$ for any $w=\mathrm{a}_{\mathrm{i} 1} \quad \mathrm{a}_{\mathrm{i} 2}$ $\ldots \mathrm{a}_{\mathrm{ik}}$ in $\mathrm{A}^{*}$. When there is no confusion, $f$ is write as $f$.

4.4.2 Theorem: Suppose $(A, Z, P, \chi, \delta)$ is a POCS. Then for any word $w$ in $A^{*}, \chi(w) \in$ $f(w)$ for some $f$ in $M f n(A, Z)$.

Proof: Suppose $(\mathrm{A}, \mathrm{Z}, \mathrm{P}, \chi, \delta)$ is a POCS.

Define $f: \mathrm{A} \rightarrow 2^{\mathrm{Z}}$ by $f\left(\mathrm{a}_{\mathrm{i}}\right)=\mathrm{Z}_{\mathrm{i}}$ for all $\mathrm{i}$.

Clearly $f$ is an element decrypt of $\operatorname{Mfn}(\mathrm{A}, \mathrm{Z})$. 
Let $w=\mathrm{a}_{\mathrm{i} 1} \mathrm{a}_{\mathrm{i} 2} \ldots \mathrm{a}_{\mathrm{ik}}$ be an element $\mathrm{A}^{*}$.

Then $\chi(w)=\mathrm{z}_{\mathrm{i} 1} \mathrm{z}_{\mathrm{i} 2} \ldots \mathrm{z}_{\mathrm{ik}}$ where $\mathrm{z}_{\mathrm{ih}} \in \mathrm{Z}_{\mathrm{ih}}$ for $1 \leq \mathrm{h} \leq \mathrm{k}$.

So $\chi(w)=\mathrm{z}_{\mathrm{i} 1} \mathrm{z}_{\mathrm{i} 2} \ldots \mathrm{z}_{\mathrm{ik}} \in \mathrm{Z}_{\mathrm{i} 1} \mathrm{Z}_{\mathrm{i} 2} \ldots \mathrm{Z}_{\mathrm{ik}}=f\left(\mathrm{a}_{\mathrm{i} 1}\right) f\left(\mathrm{a}_{\mathrm{i} 2}\right) \ldots f\left(\mathrm{a}_{\mathrm{ik}}\right)=$ $f(w)$.

\subsubsection{Definition $\left(\mathrm{Mfn}^{+}(\mathrm{D}, \mathrm{E})\right)$}

Suppose D and E are two sets. A multifunction [3], [8], [9], [10] $f$ from $D$ into $E$ is said to be a perfect multi function if $f(d)$ is nonempty for every $d$ in $D$ and $\cup_{d \in D} f(d)=E$. The set of all perfect multifunctions from $\mathrm{D}$ into $\mathrm{E}$ is denoted by $\operatorname{Mfn}^{+}(\mathrm{D}, \mathrm{E})$.

\subsubsection{Theorem: Suppose $A$ and $Z$ are two nonempty finite sets and $f \in \operatorname{Mfn}^{+}(D, E)$. Then there exists a POCS $(A, Z, P, \chi, \delta)$ such that $\chi(w) \in f(w)$ for any $w \in A^{+}$. \\ Proof: Let $f \in \operatorname{Mfn}^{+}(\mathrm{A}, \mathrm{Z})$.}

Suppose $\mathrm{P}=\left\{f\left(\mathrm{a}_{1}\right), f\left(\mathrm{a}_{2}\right), \ldots, f\left(\mathrm{a}_{\mathrm{n}}\right)\right\}$. Since $f$ is a perfect multifunction, $\mathrm{P}$ is a partition of $\mathrm{Z}$.

Let $\chi=\left\{\chi_{1}, \chi_{2}, \ldots, \chi_{\mathrm{n}}\right\}$ be a set of functions where each $\chi_{\mathrm{i}}$ : $\mathrm{N} \rightarrow \mathrm{Z}_{\mathrm{i}}$ for $1 \leq \mathrm{i} \leq \mathrm{n}$.

Let $\delta: \mathrm{Z} \rightarrow \mathrm{A}$ be defined by $\delta(\mathrm{z})=\mathrm{a}_{\mathrm{i}}$ if $\mathrm{z} \in \mathrm{Z}_{\mathrm{i}}$.

Clearly $(\mathrm{A}, \mathrm{Z}, \mathrm{P}, \chi, \delta)$ is a POCS.

Clearly for any $w$ in $\mathrm{A}^{*}, \chi(w) \in f(w)$. By the above two theorems, we understand that each element $f$ of $\operatorname{Mfn}^{+}(\mathrm{A}, \mathrm{Z})$ corresponds to a partition $\mathrm{P}$ of $\mathrm{Z}$, and each partition $\mathrm{P}$ of $\mathrm{Z}$ corresponds to an element $f$ of $\operatorname{Mfn}^{+}(\mathrm{A}, \mathrm{Z})$ By defining $\chi$ and $\delta$, we get a POCS. Since $\delta$ is a consequence of $\mathrm{P}$, we need only to define $\chi$ to get a POCS. So, each perfect multifunction $f$ represents a set of POCS. Let us denote this set by POCS $_{f}$ $(\mathrm{A}, \mathrm{Z})$. For a given input alphabet $\mathrm{A}$ and output alphabet $\mathrm{Z}$, let us denote the set of all POCS with $A$ and $Z$ as input and output alphabet by $\mathrm{P}(\mathrm{A}, \mathrm{Z})$.

4.4.5 Theorem: $P(A, Z)=\cup_{f \in M f n}{ }^{+}(A, Z) P O C S_{f}$ (A, Z).

Proof: The proof is a direct consequence.

\section{ANYLASIS OF POCS}

In this system, the output alphabet can be known if it is not a block of output alphabet, such as the one explained in example '4.2.1' If each output alphabet is a block of alphabet of fixed size (as in example 4.2.2 or 4.2.3) the size of the block can be guessed on knowing the number of alphabet in any cipher text. But if each output alphabet is variable size block of alphabet (as in example 4.2.4), then it is highly impossible to know the output alphabet. As the prediction of output alphabet itself is difficult, decoding that becomes highly impossible.

Let us suppose that the output alphabet is known and that it is not blocks of alphabet (as in example 4.2.1). In order to decrypt a given cipher, one should guess the n-partition of $\mathrm{m}$, one out of $\mathrm{x}$ possible partitions, where $\left(\begin{array}{l}m-1 \\ m-n\end{array}\right) \leq \mathrm{x} \leq$ $\left(\begin{array}{l}m-1 \\ m-n\end{array}\right)$. As $\mathrm{m}$ is very much larger than $\mathrm{n}, \mathrm{x}$ also will be large (at least $\left(\begin{array}{c}m-1 \\ m-n\end{array}\right)$. In other words, $\mathrm{K}_{2}$ is difficult of this order. Again one has to guess the initial permutation $\left(\mathrm{K}_{2}\right)$, one out of possible $\mathrm{m}$ ! permutations. So the decryption will be to guess one out of $x^{*} \mathrm{~m}$ !. So the probability of guessing the key pair $\left(\mathrm{K}_{1}, \mathrm{~K}_{2}\right)$ is $1 /\left(\mathrm{x}^{*} \mathrm{~m} !\right)$.
The probability of guessing the key pair in example 4.2.1 is $\leq 1 /(665 * 22$ ! $)=1 / 747460483972109107200$

$000=1.337863367285800494247954502632 * 10^{-24}$. This probability is achieved just with an output size of 22 . As we increasing the size of the output alphabet, the chances for decrypting a given cipher decreases.

In general the cryptosystems will be having same size of input and output alphabet. There by however the algorithm is made to encrypt, the final function that encrypts should be a bijection (one-one and onto). In other words it is simply a permutation. So if the input size is $n$, then the number of permutations is only $n !$ and the probability of guessing the key is $1 / n !$. So, to enhance the security, one needs to increase the size of the input alphabet or consider a big block of input alphabet as input alphabet. In POCS without changing the input alphabet, we achieve more secured encryption as explained by the following theorems.

\subsection{Theorem (Probability of breaking POCS)}

Suppose the size of the input alphabet is $\mathrm{n}$ and that of output alphabet is $\mathrm{kn}$ in a POCS. Then the probability of guessing the key pair $\left(\mathrm{K}_{1}, \mathrm{~K}_{2}\right)$ is approximately $(((\mathrm{k}-1) \mathrm{n}) ! \mathrm{n} !) /(\mathrm{kn}) !^{2}$.

Proof: The number of ways to choose $\mathrm{K}_{2}$ lies between $\left(\begin{array}{l}k n-1 \\ k n-n\end{array}\right)$ and $\left[\begin{array}{l}k n-1 \\ k n-n\end{array}\right]$. So the least possible value is $\left(\begin{array}{l}k n-1 \\ k n-n\end{array}\right)$ which is approximately equal to $\left(\begin{array}{c}k n \\ k n-n\end{array}\right)=\left(\begin{array}{c}k n \\ n\end{array}\right)$. So, the number of ways to select $\mathrm{K}_{2}$ can be taken as $\left(\begin{array}{c}k n \\ n\end{array}\right)$. The number of ways to choose the key $\mathrm{K}_{1}$ is $(\mathrm{kn})$ ! and hence the number of ways to choose the key pair $\left(\mathrm{K}_{1}, \mathrm{~K}_{2}\right)$ is $\left(\begin{array}{c}k n \\ n\end{array}\right)$ $(\mathrm{kn}) !=(\mathrm{kn}) !(\mathrm{kn}) ! /(((\mathrm{k}-1) \mathrm{n}) ! \mathrm{n} !)$. Hence, the probability to guess the key pair is $(((\mathrm{k}-1) \mathrm{n}) ! \mathrm{n} !) /(\mathrm{kn}) !^{2}$.

\subsection{Theorem (Probability of breaking POCS compared to general cryptosystems)}

The probability to guess the key in a general crypto system is at least $\left(\begin{array}{c}\mathrm{kn} \\ \mathrm{n}\end{array}\right)$ times of the probability to guess the key in POCS, where $\mathrm{n}$ and $\mathrm{kn}$ are respectively the sizes of input and output alphabet in POCS and kn being the size of alphabet in general crypto system.

Proof: Let us consider the alphabet (input and output) size of a general cryptosystem to be kn.

The probability to guess a key in general crypto system is $1 /$ $(\mathrm{kn})$ ! Whereas that in POCS is $(((\mathrm{k}-1) \mathrm{n}) ! \mathrm{n} !) /(\mathrm{kn}) !^{2}$. Hence the probability to guess a key in general crypto system is $(\mathrm{kn})$ ! $/(((\mathrm{k}-1) \mathrm{n}) ! \mathrm{n} !)=\left(\begin{array}{c}k n \\ n\end{array}\right)$ times that in POCS.

If we take the input alphabet as the English alphabet $\mathbf{A}$ containing 26 letters and the output alphabet as $\mathbf{A} \times\{1,2,3$, $4\}$, then the probability to break POCS is 2255101496887919458894416 times that of a general crypto system with alphabet $\mathbf{A} \times\{1,2,3,4\}$. 


\subsection{Theorem: (Probability of breaking POCS compared to general cryptosystems)}

The probability to guess the key in a block cipher [4], [5], [6], [7], [11], alphabet of size $\mathrm{n}$ and block size $\mathrm{k}$ is at least $\left(\begin{array}{c}\mathrm{n}^{\mathrm{k}} \\ \mathrm{n}\end{array}\right)$ times of the probability to guess the key in POCS with $\mathrm{n}$ and $\mathrm{n}^{\mathrm{k}}$ respectively being the sizes of input and output alphabet in POCS.

Proof: The proof is similar to the above since in block cipher, the alphabet may be treated as the set of all blocks of the alphabet. If we take the input alphabet as the English alphabet A containing 26 letters and the output alphabet as $\mathbf{A}^{\mathbf{2}}$, the strings of length 2 , then the probability to break POCS is $5.7746226578042013138408988185728 \times 10^{46}$ times that of a block cipher system with alphabet $\mathbf{A}$ having a block of size 2 .

\section{CONCLUSION}

In this paper, we generalized the four stage encryption as generated Partitioned Output Cryptosystem (GPOCS) based on multifunction concept to increase the security without altering the input alphabet size. The output alphabet may be changed so that the security is enhanced. It is also observed that the security is much higher compared to general crypto systems in use.

\section{REFERENCES}

[1] Gomatam V S Acharyulu, Sangapu V Appaji, "Analysis of Four Stage Encryption", International Journal of Research in Computer and Communication Technology, Vol. 6, Issue 4, pp. 338-339, Nov. 2012.

[2] Gomatam V S Acharyulu, Sangapu V Appaji, "Four Stage Encryption", International Journal of Research in Computer and Communication Technology, Vol. 1, Issue 4, pp. 129-132, sep. 2012.
[3] Gomatam V S Acharyulu, Matrix Representable SORings, Semigroup Forum, Vol.46, pp. 37-47, 1993.

[4] C. A. Henk, "Introduction", in Fundamentals of cryptology a professional reference and interactive tutorial van Tilborg", Eindhoven University of Technology, The Netherlands kluwer academic publishersboston/dordrecht/London,pp.3, http://hyperelliptic.org/tanja//teaching/cryptoI13/cryptodi ct.pdf.

[5] J. Hoffstein, J. Jipher, J. H. Silverman, "Intrduction to Cryptography" in Introduction to Mathematical Cryptography, Springer-Verlag, 2008, pp. 38.

[6] C. Kaufman, R. Perlman, M. Speciner, "Network Security. Upper Saddle River", NJ: Prentice Hall, 2002

[7] Moses Liskov, L. Ronald Rivest, David Wagner. "Tweakable Block Ciphers". Journal of Cryptology, 24(3), pp. July, 2011

[8] E. G. Manes, M. A. Arbib, "Algebraic Approaches to Prgram Semantics", Spriger-Verlag, New ork, Inc., 1986.

[9] E. G. Manes, D.B. Benson, "Inverse semigroup of sumordered semi ring", Semigroup Forum, Vol. 31, pp. 129 152,1985

[10] M. E. Streenstrup E., Sum-Ordered Partial Semirings, Doctoral Thesis, Department of Computer and Information Science, University of Massachusetts, 1985.

[11] William Stallings, "Cryptography and Network Security Principles and Practice Fifth Edition”, Prentice Hall, pp. 68, 198-214, 2011.

[12] Sangapu Venkata Appaji, Dr. Gomatham V S Acharyulu, "A Study of Four stage encryption: Experimental results". Accepted by IEEE International Conference on Computational Intelligence and Computing research", Coimbatore, 2014. 